\title{
Bounded and compact Toeplitz+Hankel matrices
}

\section{Article}

Accepted Version

Ehrhardt, T., Hagger, R. and Virtanen, J. (2021) Bounded and compact Toeplitz+Hankel matrices. Studia Mathematica, 2021 (260). pp. 103-120. ISSN 0039-3223 doi: https://doi.org/10.4064/sm200806-6-10 Available at https://centaur.reading.ac.uk/93267/

It is advisable to refer to the publisher's version if you intend to cite from the work. See Guidance on citing.

To link to this article DOI: http://dx.doi.org/10.4064/sm200806-6-10

Publisher: Institute of Mathematics Polish Academy of Sciences

All outputs in CentAUR are protected by Intellectual Property Rights law, including copyright law. Copyright and IPR is retained by the creators or other copyright holders. Terms and conditions for use of this material are defined in the End User Agreement.

\section{www.reading.ac.uk/centaur}

\section{CentAUR}

Central Archive at the University of Reading

Reading's research outputs online 


\title{
BOUNDED AND COMPACT TOEPLITZ+HANKEL MATRICES
}

\author{
TORSTEN EHRHARDT, RAFFAEL HAGGER, AND JANI A. VIRTANEN
}

\begin{abstract}
We show that an infinite Toeplitz + Hankel matrix $T(\varphi)+$ $H(\psi)$ generates a bounded (compact) operator on $\ell^{p}\left(\mathbb{N}_{0}\right)$ with $1 \leq p \leq$ $\infty$ if and only if both $T(\varphi)$ and $H(\psi)$ are bounded (compact). We also give analogous characterizations for Toeplitz + Hankel operators acting on the reflexive Hardy spaces. In both cases, we provide an intrinsic characterization of bounded operators of Toeplitz + Hankel form similar to the Brown-Halmos theorem. In addition, we establish estimates for the norm and the essential norm of such operators.
\end{abstract}

\section{INTRODUCTION}

In 1911, in a footnote of [22], Otto Toeplitz proved that the infinite matrix of the form $\left(\varphi_{j-k}\right)_{j, k \in \mathbb{N}_{0}}$ generates a bounded operator ${ }^{1}$ on $\ell^{2}\left(\mathbb{N}_{0}\right)$ if and only if there is a function $a \in L^{\infty}$ of the unit circle $\mathbb{T}$ such that $\hat{a}_{j}=\varphi_{j}$ for all $j \in \mathbb{Z}$, where $\hat{a}_{j}$ is the $j$-th Fourier coefficient of the function $a$. No one could have predicted the enormous impact that these and related seemingly simple matrices have had in mathematics and its applications for the past five decades, see e.g. $[3,5,6,24]$ and the references therein. Indeed, about 50 years after Toeplitz' result, the same characterization was given independently by Brown and Halmos [4, 11], who viewed Toeplitz matrices $T(a)=\left(\hat{a}_{j-k}\right)_{j, k \geq 0}$ as operators acting on the Hardy space $H^{2}$ of the unit circle $\mathbb{T}$ by the rule

$$
T(a) f=P M(a) f=P(a f),
$$

where $P$ is the orthogonal (Riesz) projection of $L^{2}$ onto $H^{2}$ and $M(a)$ is the operator of multiplication by $a$. Notice that for $j, k \geq 0$, we have

$$
\left\langle T(a) \chi_{k}, \chi_{j}\right\rangle=\left\langle a, \chi_{j-k}\right\rangle=\hat{a}_{j-k},
$$

Date: October 5, 2020.

2020 Mathematics Subject Classification. Primary 47B35; Secondary 47B37, 30H10.

Key words and phrases. Toeplitz + Hankel matrices, boundedness, compactness, sequence spaces, Hardy spaces.

${ }^{1}$ More precisely, Toeplitz showed that the finite sections of the matrix $\left(\varphi_{j-k}\right)_{j, k \geq 0}$ are uniformly bounded if and only if the same is true for the Laurent matrix $\left(\varphi_{j-k}\right)_{j, k \in \mathbb{Z}}$, which by the Banach-Steinhaus theorem characterizes boundedness. 
where $\left\{\chi_{n}: n \in \mathbb{N}_{0}\right\}$ is the standard orthonormal basis of $H^{2}$. More precisely, Brown and Halmos showed that if $A$ is a bounded linear operator on $H^{2}$ and if there are $\varphi_{n} \in \mathbb{C}$ such that $\left\langle A \chi_{k}, \chi_{j}\right\rangle=\varphi_{j-k}$ for all $j, k \geq 0$, then there is an $a \in L^{\infty}$ such that $A=T(a)$ and $\hat{a}_{n}=\varphi_{n}$ for all $n \in \mathbb{Z}$. Moreover, $\|T(a)\|=\|a\|_{\infty}$.

Since the result of Brown and Halmos, the study of Toeplitz operators has been extended to the other Hardy spaces

$$
H^{p}=\left\{f \in L^{p}: \hat{f}_{n}=0 \text { for } n<0\right\},
$$

sequence spaces $\ell^{p}\left(\mathbb{N}_{0}\right)$, and various other (analytic) function spaces. Indeed, for $1<p<\infty$, Brown and Halmos' result for $T(a)$ acting on the reflexive Hardy spaces $H^{p}$ takes the same form as in the case $p=2$ with the norm estimate

$$
\|a\|_{\infty} \leq\|T(a)\| \leq\|P\|_{B\left(L^{p}\right)}\|a\|_{\infty}
$$

where

$$
c_{p}:=\|P\|_{B\left(L^{p}\right)}=\frac{1}{\sin \frac{\pi}{p}}
$$

(see [3, Theorem 2.7] for the norm estimate and [15] for the exact value of $c_{p}$ ). In particular, $T(a)$ is bounded on $H^{p}$ if and only if $a \in L^{\infty}$. For the boundedness of Toeplitz operators on the other Hardy spaces $H^{p}$ with $0<p \leq 1$, see [16, 19, 23].

Regarding the essential norm, which is defined as

$$
\|A\|_{\mathrm{ess}}:=\inf \{\|A+K\|: K \text { compact operator }\} \text {, }
$$

it is easy to see that for $a \in L^{\infty}$

$$
\|a\|_{\infty} \leq\|T(a)\|_{\mathrm{ess}} \leq c_{p}\|a\|_{\infty}
$$

where the first inequality follows from [3, Theorem 2.30] while the other one follows from (1). As was communicated to us by E. Shargorodsky, the estimate (3) is sharp. This can be seen by considering the function $a\left(e^{i t}\right)=\sin \frac{\pi}{p} \pm i \cos \frac{\pi}{p}, \pm t \in(0, \pi)$ and using the standard Fredholm theory of piecewise continuous symbols. For further results on the essential norm of Toeplitz operators, see [21].

To define Toeplitz operators $T(a)$ on $\ell^{p}\left(\mathbb{N}_{0}\right)$ with $1 \leq p \leq \infty$, we denote by $M^{p}$ the set of all $a \in L^{1}$ for which $L(a)$, defined by $L(a) \psi:=a * \psi$ is a bounded operator on $\ell^{p}(\mathbb{Z})$. The discrete convolution $*$ is defined by

$$
(a * \psi)_{j}:=\sum_{k \in \mathbb{Z}} \hat{a}_{j-k} \psi_{k} \quad(j \in \mathbb{Z}) .
$$


For $a \in M^{p}$, we define $T(a): \ell^{p}\left(\mathbb{N}_{0}\right) \rightarrow \ell^{p}\left(\mathbb{N}_{0}\right)$ by

$$
T(a) \psi=P(a * \psi)=\left(\sum_{k=0}^{\infty} \hat{a}_{j-k} \psi_{k}\right)_{j \in \mathbb{N}_{0}}
$$

where $P\left(\ldots, x_{-1}, x_{0}, x_{1}, \ldots\right)=\left(x_{0}, x_{1}, \ldots\right)$ is the standard projection of $\ell^{p}(\mathbb{Z})$ onto $\ell^{p}\left(\mathbb{N}_{0}\right)$. In 1975 Duduchava [9] proved the following analog of the Brown-Halmos theorem when $1 \leq p<\infty$ : If $A$ is bounded on $\ell^{p}\left(\mathbb{N}_{0}\right)$ and if there is a sequence of complex numbers $\varphi_{n}$ such that $\left\langle A e_{k}, e_{j}\right\rangle=\varphi_{j-k}$ for all $j, k \in \mathbb{N}_{0}$, then there is an $a \in M^{p}$ such that $A=T(a), \hat{a}_{n}=\varphi_{n}$ for all $n \in \mathbb{Z}$, and

$$
\|T(a)\|=\|L(a)\| .
$$

Here, $\left\{e_{j}: j \in \mathbb{Z}\right\}$ denotes the standard orthonormal basis of $\ell^{2}(\mathbb{Z})$ and $\langle\cdot, \cdot\rangle$ is the dual pairing induced by the inner product on $\ell^{2}(\mathbb{Z})$. For the proof of Duduchava's result see again [3, Theorem 2.7]. To obtain the essential norm of $T(a)$ one may proceed as in [17, Section 3.7.2], which yields $\|T(a)\|_{\text {ess }}=$ $\|L(a)\|$ for $a \in M^{p}$.

The result of Duduchava can in principle be transferred to $p=\infty$ as well. However, there is one caveat. A bounded linear operator on $\ell^{\infty}\left(\mathbb{N}_{0}\right)$ is not uniquely determined by the matrix elements $\left(\left\langle A e_{k}, e_{j}\right\rangle\right)_{j, k \in \mathbb{N}_{0}}$. In other words, not every bounded linear operator on $\ell^{\infty}\left(\mathbb{N}_{0}\right)$ is given by an infinite matrix. Indeed, there are nonzero bounded linear operators $A$ on $\ell^{\infty}\left(\mathbb{N}_{0}\right)$ such that $\left\langle A e_{k}, e_{j}\right\rangle=0$ for all $j, k \in \mathbb{N}_{0}$. We refer to Chapter 1 of [17] for a discussion. As a consequence, Duduchava's result needs an additional assumption in case $p=\infty$. Namely, we need to assume that $A$ is given by an infinite matrix, that is,

$$
(A x)_{j}=\sum_{k \in \mathbb{N}_{0}}\left\langle A e_{k}, e_{j}\right\rangle x_{k}
$$

For such operators Duduchava's result is easily verified considering that the $\ell^{\infty}$-norm is the maximum absolute row sum norm in that case. Hence $M^{\infty}$ coincides with the Wiener algebra $W$ (see (5) below) and in that case

$$
\|T(a)\|_{\mathrm{ess}}=\|T(a)\|=\|L(a)\|=\sum_{j \in \mathbb{Z}}\left|\hat{a}_{j}\right|
$$

for $a \in W$.

Finally, we note that Toeplitz operators $T(a)$ are never compact unless $a=0$, which was proved in [4] for $p=2$ and can be proved similarly for the other values of $p$. This well-known result also follows as a special case of Theorem 7 (Theorem 17, respectively) below. 
Toeplitz operators are closely related to Hankel operators $H(a)$, which can be defined on Hardy spaces by setting

$$
H(a) f:=P M(a) J f=P(a J f),
$$

where $J$ is the flip operator, which is defined by $J f(t)=\bar{t} f(\bar{t})$ for $t \in \mathbb{T}$. It is easy to see that for $j, k \geq 0$,

$$
\left\langle H(a) \chi_{k}, \chi_{j}\right\rangle=\left\langle a, \chi_{j+k+1}\right\rangle=\hat{a}_{j+k+1} .
$$

The Hankel operator $H(a)$ on $\ell^{p}\left(\mathbb{N}_{0}\right)$ is defined by

$$
H(a) \varphi=\left(\sum_{k \in \mathbb{N}_{0}} \hat{a}_{j+k+1} \varphi_{k}\right)_{j \geq 0} .
$$

In 1957, Nehari [18] showed that the infinite matrix $\left(a_{j+k+1}\right)_{j, k \geq 0}$ generates a bounded operator on $\ell^{2}\left(\mathbb{N}_{0}\right)$ if and only if there is a function $b \in L^{\infty}$ such that $b_{n}=a_{n}$ for $n \in \mathbb{N}$. For $1<p<\infty$, Nehari's characterization extends to Hankel operators acting on $H^{p}$. However, there is no Nehari-type result for Hankel operators acting on $\ell^{p}\left(\mathbb{N}_{0}\right)$ for $p \notin\{1,2, \infty\}$ (see Open Problem 2.12 of [3]). For $p \in\{1, \infty\}$ one can easily see that $H(a)$ is bounded if and only if $a$ is in the Wiener algebra.

Unlike for Toeplitz operators, there is a large class of compact Hankel operators, and, more precisely, as shown by Hartman [12] in 1958 for $p=2$, the Hankel operator $H(a)$ is compact on $H^{p}$ with $1<p<\infty$ if and only if there is a continuous function $b$ such that $\hat{a}_{n}=\hat{b}_{n}$ for all $n \in \mathbb{N}$. However, as for boundedness, there is no characterization of compact Hankel operators on $\ell^{p}\left(\mathbb{N}_{0}\right)$ in terms of their symbols for $p \notin\{1,2, \infty\}$. For further details on Hankel operators, see Section 2.3 of [3] and also [20].

If $T(a)$ and $H(b)$ are both bounded, then obviously the Toeplitz + Hankel operator $T(a)+H(b)$ is bounded. It is natural to ask whether the converse statement is true. We answer this question in the affirmative and also show that the compactness of $T(a)+H(b)$ implies that both $T(a)$ and $H(b)$ are compact (and then, necessarily, $a=0$ ). Furthermore, we obtain estimates for the norm and the essential norm of bounded Toeplitz + Hankel operators. For the spectral properties of $T(a)+H(b)$ with bounded symbols, see $[1,7,8]$ and the references therein. In addition to intrinsic interest, these types of operators play a role in the asymptotic study of Toeplitz + Hankel determinants; see [2, 10] and the references therein.

Our main result is an analog of the Brown-Halmos theorem. It gives an intrinsic characterization of bounded operators of Toeplitz + Hankel form with unique symbols (see Theorem 10 and Theorem 20). 
We start with the sequence spaces $\ell^{p}\left(\mathbb{N}_{0}\right)$ by exploiting the structures of and connections between infinite Toeplitz, Laurent, Hankel, and checkerboard matrices, using operator theoretic methods, such as limit operator techniques. To deal with $p=\infty$ we use pre-adjoints which is essentially motivated by similar arguments in [17]. For the treatment of Toeplitz + Hankel operators on Hardy spaces, we consider sesquilinear (Toeplitz, Hankel etc.) forms and obtain similar characterizations. However, we only deal with the case $1<p<\infty$ in Hardy space.

\section{SEQUENCE SPACES}

Throughout this section, we assume that $1 \leq p \leq \infty$ unless stated otherwise. For an infinite set $J \subseteq \mathbb{Z}$, we say that the infinite matrix $\left(A_{j, k}\right)_{j, k \in J}$ generates a bounded linear operator on $\ell^{p}(J)$ if there is a positive constant $c$ such that for each $\varphi \in \ell^{p}(J)$, the series $\psi=\left(\psi_{j}\right)_{j \in J}$, where $\psi_{j}=$ $\sum_{k \in J} A_{j, k} \varphi_{k}$, is in $\ell^{p}(J)$ and $\|\psi\| \leq c\|\varphi\|$. In that case, there is a bounded linear operator $A$ acting on $\ell^{p}(J)$ such that

$$
A_{j, k}=\left\langle A e_{k}, e_{j}\right\rangle
$$

for all $j, k \in J$, where $\left(e_{k}\right)_{k \in J}$ is the standard basis for $\ell^{2}(J)$ and

$$
\langle\varphi, \psi\rangle=\sum_{j \in J} \varphi_{j} \overline{\psi_{j}}
$$

for $\varphi \in \ell^{p}(J)$ and $\psi \in \ell^{q}(J)$ with $1 / p+1 / q=1$. If $p<\infty$, every bounded linear operator is generated by an infinite matrix. For $p=\infty$ this is not the case, but we will only consider operators that are generated by infinite matrices. We will say that an infinite matrix is bounded if the corresponding operator is bounded. The norm of the matrix is given by the operator norm.

For the sake of completeness, we will also briefly mention bounded linear operators $A: c_{0}(J) \rightarrow \ell^{\infty}(J)$ (see Remark 3(b) below). Here $c_{0}(J) \subset \ell^{\infty}(J)$ stands for the set of sequences $\varphi=\left(\varphi_{j}\right)_{j \in J}$ for which $\lim _{|j| \rightarrow+\infty}\left|\varphi_{j}\right|=0$. In this case, every bounded linear operator is generated by an infinite matrix because sequences of finite support are dense in $c_{0}(J)$.

For a bounded sequence $\varphi=\left(\varphi_{n}\right)_{n \in \mathbb{Z}}$, let $L(\varphi)$ denote the (formal) Laurent matrix given by

$$
L(\varphi)_{j, k}=\varphi_{j-k} \quad(j, k \in \mathbb{Z}) .
$$

Similarly, we define the Toeplitz matrix $T(\varphi)$ by

$$
T(\varphi)_{j, k}=\varphi_{j-k} \quad\left(j, k \in \mathbb{N}_{0}\right)
$$


and the Hankel matrix $H(\varphi)$ by

$$
H(\varphi)_{j, k}=\varphi_{j+k+1} \quad\left(j, k \in \mathbb{N}_{0}\right) .
$$

In case these matrices define bounded operators on $\ell^{p}(\mathbb{Z})$ or $\ell^{p}\left(\mathbb{N}_{0}\right)$, respectively, we call them Laurent, Toeplitz or Hankel operators. A matrix $C$ is called a checkerboard matrix if $C_{j, k}=C_{j+1, k+1}=C_{j+1, k-1}$ for all $j, k \in \mathbb{Z}$.

We say that a sequence of matrices $A_{n}$ converges elementwise to a matrix $A$ if $\left(A_{n}\right)_{j, k}$ converges to $A_{j, k}$ for every $j, k \in J$. Similarly, a sequence $\left(\varphi_{n}\right)_{n \in \mathbb{N}}$ in $\ell^{p}(J)$ converges elementwise to $\varphi \in \ell^{p}(J)$ if $\left(\varphi_{n}\right)_{j} \rightarrow(\varphi)_{j}$ for all $j \in J$.

Lemma 1. If $\left(A_{n}\right)_{n \in \mathbb{N}}$ is a uniformly bounded sequence of matrices $A_{n}$ that converges elementwise to the matrix $A$, then $A$ generates a bounded linear operator and

$$
\|A\| \leq \sup _{n \in \mathbb{N}}\left\|A_{n}\right\|
$$

Proof. First assume $p<\infty$. Let $M:=\sup _{n \in \mathbb{N}}\left\|A_{n}\right\|$ and $\varphi \in \ell^{p}(J)$ with finite support. As $\varphi$ has finite support, the sequence $\left(A_{n} \varphi\right)_{n \in \mathbb{N}}$ converges elementwise to $A \varphi$. By Fatou's lemma, $A \varphi \in \ell^{p}(J)$ and $\|A \varphi\|_{p} \leq M\|\varphi\|_{p}$. As the elements with finite support are dense in $\ell^{p}(J), A$ extends to a bounded operator on $\ell^{p}(J)$ and the norm estimate follows.

Now assume that $p=\infty$ and consider the Hermitian transpose of the matrix $A_{n}$ defined by $\left(A_{n}^{\triangleleft}\right)_{j, k}=\overline{\left(A_{n}\right)_{k, j}}$. Notice that $A_{n}^{\triangleleft}$ generates an operator on $\ell^{1}(J)$ and is called a pre-adjoint of $A_{n}$ because $\left(A_{n}^{\triangleleft}\right)^{*}=A_{n}$. It follows $\left\|A_{n}^{\triangleleft}\right\|=\left\|A_{n}\right\|$ for all $n \in \mathbb{N}$. We can therefore apply the above for $p=1$ to the sequence $\left(A_{n}^{\triangleleft}\right)_{n \in \mathbb{N}}$ to get the required result.

We denote by $\hat{a}$ the sequence of the Fourier coefficients of $a \in L^{1}$. Note that necessarily $\hat{a} \in c_{0}(\mathbb{Z})$. For $1 \leq p \leq \infty$, the class $M^{p}$ of symbols $a \in L^{1}$ defined in the previous section can be described as the set of all $a \in L^{1}$ for which the Laurent matrix $L(\hat{a})$ generates a bounded operator on $\ell^{p}(\mathbb{Z})$. This operator is of course exactly $L(a)$ as defined in the introduction.

It is well known that for $1 \leq p \leq r \leq 2$ and $1 / p+1 / q=1$, we have $M^{p}=M^{q}$,

$$
W=M^{1} \subseteq M^{p} \subseteq M^{r} \subseteq M^{2}=L^{\infty}
$$

and

$$
\|a\|_{\infty} \leq\|L(a)\|_{r} \leq\|L(a)\|_{p} \leq\|a\|_{W}:=\sum_{j \in \mathbb{Z}}\left|\hat{a}_{j}\right|,
$$

where $W$ is the Wiener algebra of continuous functions $a$ for which $\left(\hat{a}_{j}\right)_{j \in \mathbb{Z}} \in$ $\ell^{1}(\mathbb{Z})$. From these, it follows that $M^{p} \subseteq L^{\infty}$. Each $M^{p}$ is a Banach algebra with $\|a\|_{M^{p}}:=\|L(a)\|$ for $a \in M^{p}$. These properties of the algebras $M^{p}$ 
and the following converse statement can be found in [3, Sect. 2.3-2.5], for example.

Proposition 2. Let $1 \leq p<\infty$, A be a bounded operator on $\ell^{p}(\mathbb{Z})$ and $\left\langle A e_{k}, e_{j}\right\rangle=\varphi_{j-k}$ for all $j, k \in \mathbb{Z}$, where $\left(\varphi_{j}\right)_{j \in \mathbb{Z}}$ is a sequence of complex numbers. Then there is an $a \in M^{p}$ such that $A=L(a)$ and $\hat{a}_{j}=\varphi_{j}$ for all $j \in \mathbb{Z}$.

Remarks 3. (a) Assuming that $A$ is generated by an infinite matrix, one can prove the same result for $p=\infty$ by proceeding as in Lemma 1 and using that $M^{1}=M^{\infty}=W$. Alternatively, it follows by just considering the maximum absolute row sum norm.

(b) The result remains true also for bounded linear operators $A: c_{0}(\mathbb{Z}) \rightarrow$ $\ell^{\infty}(\mathbb{Z})$. In this case, we obtain $A=L(a)$ with $a \in W$, and, as a consequence, $A$ maps $c_{0}(\mathbb{Z})$ into itself. In fact, all the results in this section remain true for bounded linear operators $A: c_{0}(J) \rightarrow \ell^{\infty}(J)$ with $J=\mathbb{Z}$ or $J=\mathbb{N}_{0}$. To see this notice that any such operator can be extended via its matrix representation to a bounded linear operator on $\ell^{\infty}(J)$. We leave the detailed verification to the reader.

For the following we will need the flip operator $J: \ell^{p}(\mathbb{Z}) \rightarrow \ell^{p}(\mathbb{Z})$, which is given by

$$
(J \varphi)_{k}=\varphi_{-k-1} .
$$

Proposition 4. For $1 \leq p \leq \infty$, let $\varphi, \psi \in \ell^{\infty}(\mathbb{Z})$ and assume $A=$ $L(\varphi)+L(\psi) J$ generates a bounded linear operator on $\ell^{p}(\mathbb{Z})$. Then both $L(\varphi)$ and $L(\psi)$ can be written as a sum of a bounded Laurent matrix and a checkerboard matrix $C$, i.e.,

$$
L(\varphi)=L(\hat{a})+C, \quad L(\psi)=L(\hat{b})-C J
$$

with $a, b \in M^{p}$, and $\max \left\{\|a\|_{M^{p}},\|b\|_{M^{p}}\right\} \leq\|A\| \leq\|a\|_{M^{p}}+\|b\|_{M^{p}}$.

Proof. Consider the standard shift $V=L\left(e_{1}\right)$. Then

$$
A-V A V=L(\varphi)+L(\psi) J-V L(\varphi) V-V L(\psi) J V=L\left(\varphi-V^{2} \varphi\right) .
$$

Similarly, $A-V^{j} A V^{j}=L\left(\varphi-V^{2 j} \varphi\right)$. Clearly, $\left\|L\left(\varphi-V^{2 j} \varphi\right)\right\| \leq 2\|A\|$. For $n \in \mathbb{N}$, consider the decomposition

$$
L(\varphi)=\frac{1}{2 n+1} \sum_{j=-n}^{n} L\left(\varphi-V^{2 j} \varphi\right)+\frac{1}{2 n+1} \sum_{j=-n}^{n} L\left(V^{2 j} \varphi\right) .
$$

Let $C_{n}:=\frac{1}{2 n+1} \sum_{j=-n}^{n} L\left(V^{2 j} \varphi\right)$. As $\varphi \in \ell^{\infty}(\mathbb{Z})$, the matrix elements of $C_{n}$ are uniformly bounded by $\|\varphi\|_{\ell^{\infty}(\mathbb{Z})}$. We can therefore choose an increasing 
sequence $\left(n_{k}\right)_{k \in \mathbb{N}}$ such that $\left(C_{n_{k}}\right)_{k \in \mathbb{N}}$ converges elementwise to some matrix $C$. As

$$
\begin{aligned}
\left|\left(C_{n_{k}}\right)_{l, m}-\left(C_{n_{k}}\right)_{l+1, m-1}\right| & =\frac{1}{2 n_{k}+1}\left|\sum_{j=-n_{k}}^{n_{k}} \varphi_{l-m-2 j}-\sum_{j=-n_{k}}^{n_{k}} \varphi_{l-m-2 j+2}\right| \\
& =\frac{1}{2 n_{k}+1}\left|\varphi_{l-m-2 n_{k}}-\varphi_{l-m+2 n_{k}+2}\right| \\
& \leq \frac{2}{2 n_{k}+1}\|\varphi\|_{\ell \infty(\mathbb{Z})},
\end{aligned}
$$

we get $(C)_{l, m}=(C)_{l+1, m-1}$ for all $l, m \in \mathbb{Z}$. Moreover, as every $C_{n}$ is a Laurent matrix, $C$ must be a Laurent matrix as well, that is, $(C)_{l, m}=$ $(C)_{l+1, m+1}$. This implies that $C$ is a checkerboard matrix. Furthermore, the sequence $\left(L(\varphi)-C_{n_{k}}\right)_{k \in \mathbb{N}}$ converges elementwise to $L(\varphi)-C$. As

$$
\left\|L(\varphi)-C_{n_{k}}\right\|=\left\|\frac{1}{2 n_{k}+1} \sum_{j=-n_{k}}^{n_{k}} L\left(\varphi-V^{2 j} \varphi\right)\right\| \leq 2\|A\|
$$

for all $k \in \mathbb{N}$, the limit $L(\varphi)-C$ is again bounded by Lemma 1 . Moreover, since both $L(\varphi)$ and $C$ are Laurent matrices, $L(\varphi)-C$ is again a Laurent matrix. We conclude that $L(\varphi)-C=L(\hat{a})$ with $a \in M^{p}$ by Proposition 2 .

For $L(\psi)$, we use $J^{2}=I$ to obtain

$$
L(\psi)+C J=A J-L(\varphi) J+C J=(A-L(\hat{a})) J .
$$

Here, the left hand side is a Laurent matrix and the right hand side is bounded. Therefore, it is equal to $L(\hat{b})$ for some $b \in M^{p}$ again by Proposition 2.

To obtain the norm estimate, observe that $A=L(\hat{a})+L(\hat{b}) J$. As $\hat{b} \in$ $c_{0}(\mathbb{Z})$, the sequence

$$
V^{-n} A V^{n}=L(\hat{a})+L\left(e_{-2 n} * \hat{b}\right) J
$$

converges elementwise to $L(\hat{a})$. By Lemma 1 this implies that $\|a\|_{M^{p}}=$ $\|L(\hat{a})\| \leq\|A\|$. Now consider $A J=L(\hat{a}) J+L(\hat{b})$ instead of $A$ to get $\|b\|_{M^{p}} \leq$ $\|A\|$. The upper estimate is trivial.

Theorem 5. For $1 \leq p \leq \infty$, let $\varphi \in \ell^{\infty}(\mathbb{Z}), \psi \in \ell^{\infty}(\mathbb{N})$ and assume that $A:=T(\varphi)+H(\psi)$ generates a bounded operator on $\ell^{p}\left(\mathbb{N}_{0}\right)$. Then $\varphi=$ $\varphi_{1}+\varphi_{2}$, where $\varphi_{1}$ is the Fourier sequence of an $M^{p}$-function and $T\left(\varphi_{2}\right)$ is a checkerboard matrix. Moreover, $\psi=\psi_{1}+\psi_{2}$, where $H\left(\psi_{1}\right)$ is a bounded Hankel matrix with $\psi_{1} \in c_{0}(\mathbb{N})$ and $H\left(\psi_{2}\right)=-T\left(\varphi_{2}\right)$. Both decompositions are unique. 
Proof. Let $P: \ell^{p}(\mathbb{Z}) \rightarrow \ell^{p}\left(\mathbb{N}_{0}\right)$ be the canonical projection and $P^{*}: \ell^{p}\left(\mathbb{N}_{0}\right) \rightarrow$ $\ell^{p}(\mathbb{Z})$ the inclusion. For $n \in \mathbb{N}$, consider $V^{-n} P^{*} A P V^{n}$ and choose an increasing sequence $\left(n_{k}\right)_{k \in \mathbb{N}}$ such that

$$
\left(V^{-n_{k}} P^{*} A P V^{n_{k}}\right)_{k \in \mathbb{N}}
$$

converges elementwise. Clearly, the limit is of the form $L(\varphi)+L(\nu) J$ with $\nu \in l^{\infty}(\mathbb{Z})$. Since

$$
\left\|V^{-n_{k}} P^{*} A P V^{n_{k}}\right\| \leq\|A\|
$$

for all $k \in \mathbb{N}, L(\varphi)+L(\nu) J$ is again a bounded operator (see Lemma 1) and we can apply Proposition 4 . Therefore, $L(\varphi)=L\left(\varphi_{1}\right)+L\left(\varphi_{2}\right)$, where $L\left(\varphi_{1}\right)$ is bounded and $L\left(\varphi_{2}\right)$ is a checkerboard matrix. This implies that $T\left(\varphi_{1}\right)$ is bounded, $\varphi_{1}$ is the Fourier sequence of an $M^{p}$-function by Proposition 4 and $T\left(\varphi_{2}\right)$ is a checkerboard matrix. Using $H(\psi)=(T(\varphi)+H(\psi))-T\left(\varphi_{1}\right)-$ $T\left(\varphi_{2}\right)$, we get the decomposition for $\psi$. The uniqueness follows from the obvious fact that the only bounded checkerboard matrix is 0 . Finally notice that if $H\left(\psi_{1}\right)$ is a bounded Hankel matrix, then $\psi_{1} \in c_{0}(\mathbb{N})$, which can be seen by considering the first row or column.

Remarks 6. (a) As a consequence, $A=T(\hat{a})+H\left(\psi_{1}\right)$ with $a \in M^{p}$ and $\psi_{1} \in c_{0}(\mathbb{N})$ where $H\left(\psi_{1}\right)$ is a bounded Hankel matrix.

(b) Note that the precise condition for the boundedness of Hankel operators on $\ell^{p}\left(\mathbb{N}_{0}\right), p \notin\{1,2, \infty\}$ is unknown (cf. [3, Open problem 2.12]). For $p=2$ we can use that $H\left(\psi_{1}\right)$ is unitarily equivalent to a Hankel operator $H(a)$ on $H^{2}$ and therefore $\psi_{1}$ is the (positive) Fourier sequence of a function $a \in L^{\infty}\left[3\right.$, Theorem 2.11]. For $p \in\{1, \infty\}$ it is easily seen that $H\left(\psi_{1}\right)$ is bounded if and only if $\psi_{1} \in \ell^{1}(\mathbb{Z})$. In that case $\psi_{1}$ is the Fourier sequence of a function in the Wiener algebra and $H\left(\psi_{1}\right)$ is also compact.

Theorem 7. For $1 \leq p \leq \infty$, let $A=T(\hat{a})+H\left(\psi_{1}\right)$ where $a \in M^{p}$ and $\psi_{1} \in c_{0}(\mathbb{N})$ such that $H\left(\psi_{1}\right)$ generates a bounded Hankel matrix on $\ell^{p}\left(\mathbb{N}_{0}\right)$. Then, for any compact operator $K$,

$$
\max \left\{\|a\|_{M^{p}}, \frac{1}{2}\left\|H\left(\psi_{1}\right)+K\right\|\right\} \leq\|A+K\| \leq\|a\|_{M^{p}}+\left\|H\left(\psi_{1}\right)+K\right\| .
$$

Proof. Proceeding as in the last part of the proof of Proposition 4, we observe that the elementwise limit of $V^{-n} P^{*} A P V^{n}$ is $L(\hat{a})$ since $\psi_{1} \in c_{0}(\mathbb{N})$. Furthermore, the elementwise limit of $V^{-n} P^{*} K P V^{n}$ is zero. Indeed, this is clear for $1<p<\infty$ as the operators $V^{ \pm n} \rightarrow 0$ weakly as $n \rightarrow \infty$. For $p=\infty$ notice that, for fixed $k$, the sequence $V^{n} e_{k} \rightarrow 0$ converges weakly on the space $\ell^{\infty}(\mathbb{Z})$, hence $\left\|K P V^{n} e_{k}\right\| \rightarrow 0$. Therefore $\left\langle V^{-n} P^{*} K P V^{n} e_{k}, e_{j}\right\rangle \rightarrow 0$ for each fixed $k, j \in \mathbb{Z}$. The case $p=1$ is reduced to the case $p=$ 
$\infty$ by passing to the adjoint and noticing that $\left\langle V^{-n} P^{*} K P V^{n} e_{k}, e_{j}\right\rangle=$ $\left\langle e_{k}, V^{-n} P^{*} K^{*} P V^{n} e_{j}\right\rangle \rightarrow 0$ by the previous argument.

Therefore, $V^{-n} P^{*}(A+K) P V^{n} \rightarrow L(\hat{a})$ elementwise, and from Lemma 1 we obtain $\|a\|_{M^{p}}=\|L(\hat{a})\| \leq\|A+K\|$. To proceed consider

$$
\left\|H\left(\psi_{1}\right)+K\right\| \leq\|A+K\|+\|T(\hat{a})\| \leq\|A+K\|+\|a\|_{M^{p}} \leq 2\|A+K\|,
$$

which settles the lower estimate. The upper estimate is trivial.

Corollary 8. Let $1 \leq p \leq \infty, \varphi \in \ell^{\infty}(\mathbb{Z}), \psi \in \ell^{\infty}(\mathbb{N})$ and assume that $A:=T(\varphi)+H(\psi)$ generates a compact operator on $\ell^{p}\left(\mathbb{N}_{0}\right)$. Then $T(\varphi)$ is a checkerboard matrix and $\psi=\psi_{1}+\psi_{2}$, where $H\left(\psi_{1}\right)$ is a compact Hankel matrix with $\psi_{1} \in c_{0}(\mathbb{N})$ and $H\left(\psi_{2}\right)=-T(\varphi)$. Moreover, the decomposition of $\psi$ is unique.

Proof. We know from Theorem 5 that we can decompose $\varphi=\hat{a}+\varphi_{2}$ with $a \in M^{p}$ and $\psi=\psi_{1}+\psi_{2}$ such that $H\left(\psi_{2}\right)=-T\left(\varphi_{2}\right)$ is checkerboard and $H\left(\psi_{1}\right)$ is bounded. So, $A=T(\hat{a})+H\left(\psi_{1}\right)$. Now apply Theorem 7 with $K=-A$ to conclude that $a=0$ and $H\left(\psi_{1}\right)=-K$ is compact.

Remark 9. As for the boundedness, we remark that the precise condition for the compactness of Hankel operators on $\ell^{p}\left(\mathbb{N}_{0}\right), p \notin\{1,2, \infty\}$ is unknown (cf. [3, Open problem 2.56]). For $p=2$ the Hankel operator $H\left(\psi_{1}\right)$ is compact if and only if $\psi$ is the (positive) Fourier sequence of a function $a \in C(\mathbb{T})+\overline{H^{\infty}}$; see [3, Theorem 2.54]. For $p \in\{1, \infty\}$ see Remark 6 .

Finally, we add a characterization involving displacement relations that also nicely summarizes this section. Statement (i) provides an intrinsic characterization of bounded operators of Toeplitz + Hankel form. The underlying "displacement transform" shown in (6) is well-known in the theory of finite Toeplitz + Hankel matrices, see $[13,14]$.

Theorem 10. For $1 \leq p \leq \infty$, the following statements are equivalent:

(i) $A$ is a bounded linear operator on $\ell^{p}\left(\mathbb{N}_{0}\right)$ with matrix representation $A=\left(A_{j, k}\right)_{j, k \in \mathbb{N}_{0}}$ satisfying the displacement relations

$$
A_{j-1, k}+A_{j+1, k}-A_{j, k-1}-A_{j, k+1}=0 \quad(j, k \in \mathbb{N}) .
$$

(ii) $A$ is a bounded linear operator on $\ell^{p}\left(\mathbb{N}_{0}\right)$ whose matrix representation can be written as $T(\varphi)+H(\psi)$ for some $\varphi \in \ell^{\infty}(\mathbb{Z})$ and $\psi \in \ell^{\infty}(\mathbb{N})$.

(iii) $A=T(\hat{a})+H(\varrho)$ for some $a \in M^{p}$ and $\varrho \in c_{0}(\mathbb{N})$ such that $H(\varrho)$ is bounded on $\ell^{p}\left(\mathbb{N}_{0}\right)$. 
Furthermore, the function $a$ and the sequence $\varrho$ in (iii) are uniquely determined, and

$$
\begin{aligned}
\|T(\hat{a})+H(\varrho)\| & \simeq \max \left\{\|a\|_{M^{p}},\|H(\varrho)\|\right\}, \\
\|T(\hat{a})+H(\varrho)\|_{\mathrm{ess}} & \simeq \max \left\{\|a\|_{M^{p}},\|H(\varrho)\|_{\mathrm{ess}}\right\},
\end{aligned}
$$

where " $\simeq "$ stands for equivalence of norms.

Proof. The implications (iii) $\Rightarrow($ ii $) \Rightarrow$ (i) are obvious.

(i) $\Rightarrow($ ii): Choose

$$
\psi_{j}= \begin{cases}A_{\frac{j-1}{2}, \frac{j-1}{2}} & \text { if } j \text { is odd } \\ A_{\frac{j-2}{2}, \frac{j}{2}} & \text { if } j \text { is even. }\end{cases}
$$

Let $T=A-H(\psi)$. It remains to show that $T$ is a Toeplitz matrix. Assume that $j+k$ is even. Iterating the displacement relations gives

$$
A_{j+1, k+1}-A_{j, k}=A_{j, k+2}-A_{j-1, k+1}=\ldots=A_{j-n+1, k+n+1}-A_{j-n, k+n}
$$

for all $n \in\{-k, \ldots, j\}$. Using (7) with $n=\frac{j-k}{2}$, we get

$$
\begin{aligned}
T_{j+1, k+1}-T_{j, k} & =A_{j+1, k+1}-A_{j, k}-\psi_{j+k+3}+\psi_{j+k+1} \\
& =A_{j+1, k+1}-A_{j, k}-A_{\frac{j+k}{2}+1, \frac{j+k}{2}+1}+A_{\frac{j+k}{2}, \frac{j+k}{2}} \\
& =0 .
\end{aligned}
$$

Similarly, if $j+k$ is odd, then

$$
\begin{aligned}
T_{j+1, k+1}-T_{j, k} & =A_{j+1, k+1}-A_{j, k}-\psi_{j+k+3}+\psi_{j+k+1} \\
& =A_{j+1, k+1}-A_{j, k}-A_{\frac{j+k+1}{2}, \frac{j+k+3}{2}}+A_{\frac{j+k-1}{2}, \frac{j+k+1}{2}} \\
& =0,
\end{aligned}
$$

using (7) with $n=\frac{j-k+1}{2}$. Hence $T=T(\varphi)$ for some bi-infinite sequence $\varphi=\left(\varphi_{j}\right)_{j \in \mathbb{Z}}$. Note that $\psi$ and $\varphi$ are bounded by construction because $\left|A_{j, k}\right| \leq\|A\|$ for all $j, k \in \mathbb{N}_{0}$.

(ii) $\Rightarrow$ (iii): From Theorem 5 we conclude that $A=T(\varphi)+H(\psi)$ can be written as $A=T(\hat{a})+H(\varrho)$ with $a \in M^{p}, \varrho \in c_{0}(\mathbb{N})$ and $H(\varrho)$ being bounded.

The uniqueness of $a$ and $\varrho$ follows from the uniqueness in Theorem 5, and the statements about the norms follow from Theorem 7 .

We also have the following analogue for operators on $\ell^{p}(\mathbb{Z})$.

Proposition 11. For $1 \leq p \leq \infty$, let $A$ be a bounded linear operator on $\ell^{p}(\mathbb{Z})$ with matrix representation $A=\left(A_{j, k}\right)_{j, k \in \mathbb{Z}}$ and

$$
A_{j-1, k}+A_{j+1, k}-A_{j, k-1}-A_{j, k+1}=0 \quad(j, k \in \mathbb{Z}) .
$$


Then $A=L(\hat{a})+L(\hat{b}) J$ for some $a, b \in M^{p}$ and this decomposition is unique.

Proof. Analogous to Theorem 10.

\section{HARDY SPACES}

In this section we consider Toeplitz + Hankel operators on Hardy spaces $H^{p}(1<p<\infty)$ and prove analogous characterizations for their boundedness and compactness as in the previous section. Unfortunately, we are currently unable to deal with Toeplitz + Hankel operators on the Hardy space $H^{1}$ but conjecture that also in this case the boundedness (compactness) of $T(a)+H(b)$ implies that both $T(a)$ and $H(b)$ are bounded (compact). We will also consider multiplication operators with flip $M(a)+M(c) J$ on $L^{p}$. The corresponding results are valid for $1 \leq p<\infty$.

For $j \in \mathbb{Z}$, define $\chi_{j}: \mathbb{T} \rightarrow \mathbb{C}$ by $\chi_{j}(z)=z^{j}$ and let $U^{j}=M\left(\chi_{j}\right)$.

Lemma 12. Let $a: \mathbb{T} \rightarrow \mathbb{C}$ be a measurable function and assume that there is a constant $C>0$ such that

$$
\left\|a\left(1-\chi_{2 l}\right)\right\|_{\infty} \leq C
$$

for all $l \in \mathbb{N}$. Then $a \in L^{\infty}$ and $\|a\|_{\infty} \leq C / 2$.

Proof. Let $z \in \mathbb{T}$ such that $z^{2 l} \neq 1$ for every $l \in \mathbb{Z} \backslash\{0\}$. Then $\left\{z^{2 l}: l \in \mathbb{Z}\right\}$ is dense in $\mathbb{T}$, and the assumption implies

$$
|a(z)| \leq \inf _{l \in \mathbb{Z}} \frac{C}{\left|1-z^{2 l}\right|}=\frac{C}{\sup _{l \in \mathbb{Z}}\left|1-z^{2 l}\right|}=\frac{C}{2} .
$$

Since almost all $z \in \mathbb{T}$ satisfy the afore-mentioned condition, the assertion follows.

For measurable functions $f, g: \mathbb{T} \rightarrow \mathbb{C}$ such that $f \cdot g \in L^{1}$ we define

$$
\langle f, g\rangle:=\frac{1}{2 \pi} \int_{\mathbb{T}} f\left(e^{i \theta}\right) \overline{g\left(e^{i \theta}\right)} \mathrm{d} \theta .
$$

For $a \in L^{\infty}$ denote by $M(a)$ the operator of multiplication by $a$. We recall the following useful characterization of multiplication operators on $L^{p}$. While it is stated for $1<p<\infty$ in [3, Proposition 2.2], its proof remains valid also for $p=1$.

Proposition 13. For $1 \leq p<\infty$, let $A$ be a bounded linear operator on $L^{p}$, and suppose that there are complex numbers $\varphi_{j}$ such that

$$
\left\langle A \chi_{k}, \chi_{j}\right\rangle=\varphi_{j-k}
$$


for $j, k \in \mathbb{Z}$, that is, $A$ is of Laurent structure. Then there is an $a \in L^{\infty}$ such that $A=M(a), a_{j}=\varphi_{j}$ for all $j \in \mathbb{Z}$, and

$$
\|M(a)\|=\|a\|_{\infty} .
$$

Proposition 14. For $1 \leq p<\infty$, let $A$ be a bounded linear operator on $L^{p}$ and assume that

$$
\left\langle A \chi_{k-1}, \chi_{j}\right\rangle-\left\langle A \chi_{k}, \chi_{j-1}\right\rangle-\left\langle A \chi_{k}, \chi_{j+1}\right\rangle+\left\langle A \chi_{k+1}, \chi_{j}\right\rangle=0
$$

for all $j, k \in \mathbb{Z}$. Then $A=M(a)+M(c) J$ for some $a, c \in L^{\infty}$ and this decomposition is unique. Furthermore,

$$
\max \left\{\|a\|_{\infty},\|c\|_{\infty}\right\} \leq\|A\| \leq\|a\|_{\infty}+\|c\|_{\infty} .
$$

Proof. Observe

$$
\begin{aligned}
\left\langle(A-U A U) \chi_{k-1}, \chi_{j}\right\rangle & =\left\langle A \chi_{k-1}, \chi_{j}\right\rangle-\left\langle A \chi_{k}, \chi_{j-1}\right\rangle \\
& =\left\langle A \chi_{k}, \chi_{j+1}\right\rangle-\left\langle A \chi_{k+1}, \chi_{j}\right\rangle \\
& =\left\langle(A-U A U) \chi_{k}, \chi_{j+1}\right\rangle
\end{aligned}
$$

for all $j, k \in \mathbb{Z}$, i.e., $A-U A U$ has Laurent structure and is bounded, hence has to be equal to $M\left(\tilde{a}_{1}\right)$ for a bounded symbol $\tilde{a}_{1}$ with $\left\|\tilde{a}_{1}\right\|_{\infty} \leq 2\|A\|$ by Proposition 13. More generally, for $l \in \mathbb{N}$, consider

$$
A-U^{l} A U^{l}=\sum_{j=0}^{l-1} U^{j}(A-U A U) U^{j}=\sum_{j=0}^{l-1} M\left(\chi_{j}\right) M\left(\tilde{a}_{1}\right) M\left(\chi_{j}\right)=M\left(\tilde{a}_{l}\right)
$$

with $\tilde{a}_{l}(z):=\left(1+z^{2}+\cdots+z^{2(l-1)}\right) \tilde{a}_{1}(z)=\frac{1-z^{2 l}}{1-z^{2}} \tilde{a}_{1}(z)$, which has to satisfy the estimate $\left\|\tilde{a}_{l}\right\|_{\infty} \leq 2\|A\|$ again by Proposition 13. Defining

$$
a(z):=\frac{\tilde{a}_{1}(z)}{1-z^{2}}=\frac{\tilde{a}_{l}(z)}{1-z^{2 l}}
$$

for almost every $z \in \mathbb{T}$, it follows that

$$
\left\|a\left(1-\chi_{2 l}\right)\right\|_{\infty}=\left\|\tilde{a}_{l}\right\|_{\infty} \leq 2\|A\| .
$$

for every $l \in \mathbb{N}$. By Lemma 12, $a$ is essentially bounded and $\|a\|_{\infty} \leq\|A\|$.

Moreover,

$$
M(a)-U M(a) U=M\left(a\left(1-\chi_{2}\right)\right)=M\left(\tilde{a}_{1}\right)=A-U A U .
$$

Now consider $B:=(A-M(a)) J$, which is bounded on $L^{p}$. Then

$$
U B U^{-1}=U(A-M(a)) J U^{-1}=U(A-M(a)) U J=(A-M(a)) J=B
$$

by (10), i.e., $B$ has Laurent structure. This means that there is a $c \in L^{\infty}$ such that $B=M(c)$. Hence, $A=M(a)+M(c) J$.

To show uniqueness assume that there are $\tilde{a}, \tilde{c} \in L^{\infty}$ such that

$$
M(\tilde{a})+M(\tilde{c}) J=A=M(a)+M(c) J .
$$


Then $M(a-\tilde{a})=M(\tilde{c}-c) J$ and hence

$$
\begin{aligned}
\left\langle a-\tilde{a}, \chi_{k}\right\rangle & =\left\langle M(a-\tilde{a}) \chi_{0}, \chi_{k}\right\rangle=\left\langle M(\tilde{c}-c) J \chi_{0}, \chi_{k}\right\rangle \\
& =\left\langle M(\tilde{c}-c) \chi_{-1}, \chi_{k}\right\rangle=\left\langle M(\tilde{c}-c) \chi_{0}, \chi_{k+1}\right\rangle \\
& =\left\langle M(\tilde{c}-c) J \chi_{-1}, \chi_{k+1}\right\rangle=\left\langle M(a-\tilde{a}) \chi_{-1}, \chi_{k+1}\right\rangle \\
& =\left\langle M(a-\tilde{a}) \chi_{0}, \chi_{k+2}\right\rangle=\left\langle a-\tilde{a}, \chi_{k+2}\right\rangle
\end{aligned}
$$

for all $k \in \mathbb{Z}$. It follows $a=\tilde{a}$ and $c=\tilde{c}$ almost everywhere by the RiemannLebesgue lemma.

Regarding the norm estimates, we have already shown $\|a\|_{\infty} \leq\|A\|$. By considering the operator $A J=M(a) J+M(c)$ instead of $A=M(a)+M(c) J$ and noting that it also satisfies (9), we can repeat the arguments at the beginning of the proof. Correspondingly, we conclude that $A J=M(\tilde{a})+$ $M(\tilde{c}) J$ with certain $\tilde{a}, \tilde{c} \in L^{\infty}$ and $\|\tilde{a}\|_{\infty} \leq\|A J\|=\|A\|$. The uniqueness of the representation entails that $c=\tilde{a}, a=\tilde{c}$, and thus $\|c\|_{\infty} \leq\|A\|$. The upper norm estimates are obvious.

Remark 15. It is straightforward to verify that, conversely, an operator $A=M(a)+M(c) J$ with $a, c \in L^{\infty}$ satisfies condition (9). The norm of such an operator can even be evaluated exactly. This uses the decomposition of $L^{p}$ into the direct sum $L^{p}\left(\mathbb{T}_{+}\right) \dot{+} J L^{p}\left(\mathbb{T}_{+}\right)$, where $\mathbb{T}_{+}=\{z \in \mathbb{T}: \operatorname{Im}(z)>0\}$. The operator can then be identified with a block multiplication operator with $2 \times 2$ matrix-valued symbol. Indeed,

$$
\|A\|_{B\left(L^{p}\right)}=\operatorname{ess-sup}_{z \in \mathbb{T}_{+}}\left\|\left(\begin{array}{cc}
a(z) & c(z) \\
c(\bar{z}) & a(\bar{z})
\end{array}\right)\right\|_{p}
$$

where $\|\cdot\|_{p}$ stands for the $p$-norm of a matrix. We leave the detailed verification to the reader.

In the following we will consider sesquilinear forms $(\cdot, \cdot): \mathcal{D}_{+} \times \mathcal{D}_{+} \rightarrow \mathbb{C}$ with domain

$$
\mathcal{D}_{+}:=\operatorname{span}\left\{\chi_{j}: j \in \mathbb{N}_{0}\right\} .
$$

Every densely defined operator $A: H^{p} \rightarrow H^{p}$ with domain $\mathcal{D}(A) \supset \mathcal{D}_{+}$ induces a form via $(\cdot, \cdot)_{A}:=\langle A \cdot, \cdot\rangle$. To keep the notation slick, we will identify $A$ with $(\cdot, \cdot)_{A}$ if $(\cdot, \cdot)_{A}$ is the form induced by $A$. Conversely, if $(\cdot, \cdot)_{A}$ is a form, we will write $\langle A \cdot, \cdot\rangle:=(\cdot, \cdot)_{A}$ even if the form is not induced by an operator $A$. Addition of forms and right multiplication with operators that leave $\mathcal{D}_{+}$invariant are defined in the obvious way.

A form $T$ is called a Toeplitz form or form of Toeplitz type if $\left\langle T \chi_{k}, \chi_{j}\right\rangle=$ $\left\langle T \chi_{k+1}, \chi_{j+1}\right\rangle$ for all $j, k \in \mathbb{N}_{0}$. A form $H$ is called a Hankel form or form of 
Hankel type if $\left\langle H \chi_{k+1}, \chi_{j}\right\rangle=\left\langle H \chi_{k}, \chi_{j+1}\right\rangle$ for all $j, k \in \mathbb{N}_{0}$. If there exists a function $a \in L^{1}$ such that for $j, k \in \mathbb{N}_{0}$

$$
\left\langle T \chi_{k}, \chi_{j}\right\rangle=\left\langle a, \chi_{j-k}\right\rangle \quad \text { or } \quad\left\langle H \chi_{k}, \chi_{j}\right\rangle=\left\langle a, \chi_{1+j+k}\right\rangle,
$$

respectively, then we say that the Toeplitz or Hankel form is induced by $a \in L^{1}$ and we will write $T=T(a)$ and $H=H(a)$. If a form is both a Toeplitz and a Hankel form we call it a checkerboard form. Clearly, the only bounded checkerboard form is 0 .

Theorem 16. For $1<p<\infty$, let $T$ and $H$ be forms of Toeplitz and Hankel type, respectively, and assume that $A=T+H$ is a bounded operator on $H^{p}$. Then

$$
T=T_{1}+C \quad \text { and } \quad H=H_{1}-C,
$$

where $T_{1}$ is a bounded Toeplitz operator, $H_{1}$ is a bounded Hankel operator, and $C$ is a checkerboard form. Both decompositions are unique.

Furthermore, $T_{1}=T(a)$ and $H_{1}=H(b)$ for some $a, b \in L^{\infty}$, where the functions $a$ and $P\left(\chi_{-1} b\right)$ are uniquely determined.

Proof. Let $P: L^{p} \rightarrow H^{p}$ be the Riesz projection and $P^{*}: H^{p} \rightarrow L^{p}$ be the inclusion. As the operators $\left(U^{-n} P^{*} A P U^{n}\right)_{n \in \mathbb{N}}$ have a uniformly bounded norm, a standard argument shows that one can choose an increasing sequence $\left(n_{l}\right)_{l \in \mathbb{N}}$ such that the sequence $\left(\left\langle U^{-n_{l}} P^{*} A P U^{n_{l}} \chi_{k}, \chi_{j}\right\rangle\right)_{l \in \mathbb{N}}$ converges for every $j, k \in \mathbb{Z}$. As a consequence, the sequence $\left(U^{-n_{l}} P^{*} A P U^{n_{l}}\right)_{l \in \mathbb{N}}$ converges weakly to some bounded linear operator $B: L^{p} \rightarrow L^{p}$.

For $j, k \in \mathbb{N}_{0}$ let $t_{j-k}:=\left\langle T \chi_{k}, \chi_{j}\right\rangle$ and $h_{j+k+1}:=\left\langle H \chi_{k}, \chi_{j}\right\rangle$. Then

$$
\lim _{l \rightarrow \infty}\left\langle U^{-n_{l}} P^{*} T P U^{n_{l}} \chi_{k}, \chi_{j}\right\rangle=\lim _{l \rightarrow \infty}\left\langle T \chi_{k+n_{l}}, \chi_{j+n_{l}}\right\rangle=t_{j-k}
$$

for all $j, k \in \mathbb{Z}$. It follows that the limits $\lim _{l \rightarrow \infty}\left\langle U^{-n_{l}} P^{*} H P U^{n_{l}} \chi_{k}, \chi_{j}\right\rangle$ also exist and

$\lim _{l \rightarrow \infty}\left\langle U^{-n_{l}} P^{*} H P U^{n_{l}} \chi_{k}, \chi_{j}\right\rangle=\lim _{l \rightarrow \infty}\left\langle H \chi_{k+n_{l}}, \chi_{j+n_{l}}\right\rangle=\lim _{l \rightarrow \infty} h_{j+k+2 n_{l}+1}=: \tilde{h}_{j+k}$ for all $j, k \in \mathbb{Z}$. Combining the previous two equations, we get

$$
\left\langle B \chi_{k}, \chi_{j}\right\rangle=t_{j-k}+\tilde{h}_{j+k},
$$

and therefore $B$ satisfies (9). Hence, by Proposition 14, $B=M(a)+M(c) J$ for some $a, c \in L^{\infty}$. In particular, for $j, k \in \mathbb{N}_{0}$,

$$
t_{j-k}-\left\langle T(a) \chi_{k}, \chi_{j}\right\rangle=t_{j-k}-\left\langle M(a) \chi_{k}, \chi_{j}\right\rangle=\left\langle M(c) J \chi_{k}, \chi_{j}\right\rangle-\tilde{h}_{j+k} .
$$

The left-hand side is invariant under $j \mapsto j+1, k \mapsto k+1$. The right-hand side is invariant under $j \mapsto j-1, k \mapsto k+1$. It follows that $C:=T-T(a)$ is of checkerboard type and $T_{1}:=T(a)$ is bounded. Consequently, $H_{1}:=$ 
$H+C=H+T-T_{1}=A-T_{1}$ is a Hankel form and bounded on $H^{p}$. This yields the desired decompositions. The uniqueness follows from the fact that a checkerboard form is necessarily unbounded or zero.

Finally, the characterization of bounded Hankel operators [3, Theorem 2.11] implies that $H_{1}=H(b)$ with some (non-unique) $b \in L^{\infty}$, and we know already $T_{1}=T(a)$. Clearly, the functions $a$ and $P\left(\chi_{-1} b\right)$ are uniquely determined as their Fourier coefficients are given by $T_{1}$ and $H_{1}$.

The reasoning in the previous proof breaks down in the case $p=1$. Indeed, as $P$ is not bounded on $H^{1}$, we cannot conclude $a \in L^{\infty}$ as easily in the first part and even if we had $a \in L^{\infty}$, the corresponding Toeplitz operator $T(a)$ needs not be bounded on $H^{1}$. As mentioned at the beginning of this section, we conjecture that for $p=1$ the boundedness of $T(a)$ can be shown by different means.

Theorem 17. For $1<p<\infty$, consider the bounded linear operator $A=$ $T(a)+H(b)$ on $H^{p}$ with $a, b \in L^{\infty}$. Then, for any compact operator $K$,

$$
\max \left\{\|a\|_{\infty}, \frac{1}{1+c_{p}}\|H(b)+K\|\right\} \leq\|A+K\| \leq c_{p}\|a\|_{\infty}+\|H(b)+K\|
$$

where $c_{p}=\|P\|_{B\left(L^{p}\right)}$.

We recall that the constant $c_{p}$ is equal to $\frac{1}{\sin \left(\frac{\pi}{p}\right)}($ see $(2))$.

Proof. In the proof of Theorem 16 it was shown that $B=M(a)+M(c) J$ is a bounded linear operator on $L^{p}$, which is the weak limit of the sequence of operators $\left(U^{-n_{l}} P^{*} A P U^{n_{l}}\right)_{l \in \mathbb{N}}$. As $\left(U^{n}\right)_{n \in \mathbb{N}}$ converges weakly to 0 and $K$ is compact, $\left(K P U^{n}\right)_{n \in \mathbb{N}}$ converges strongly to 0 . It follows that $\left(U^{-n} P^{*} K P U^{n}\right)_{n \in \mathbb{N}}$ converges strongly to 0 as well. Therefore, $B$ is the weak limit of the sequence $\left(U^{-n_{l}} P^{*}(A+K) P U^{n_{l}}\right)_{l \in \mathbb{N}}$, and this implies that for each Laurent polynomial $f$,

$$
\begin{aligned}
\|B f\|_{L^{p}} & \leq \liminf _{l \rightarrow \infty}\left\|U^{-n_{l}} P^{*}(A+K) P U^{n_{l}} f\right\|_{L^{p}} \\
& \leq\|A+K\|_{B\left(H^{p}\right)} \liminf _{l \rightarrow \infty}\left\|P U^{n_{l}} f\right\|_{H^{p}}=\|A+K\|_{B\left(H^{p}\right)}\|f\|_{L^{p}} .
\end{aligned}
$$

We deduce that $\|B\| \leq\|A+K\|$, and combining this with the norm estimate $\|a\| \leq\|B\|$ established in Proposition 14 we obtain $\|a\| \leq\|A+K\|$, which is part of the lower estimate. For the upper estimate notice that $\|T(a)\| \leq c_{p}\|a\|$. Furthermore,

$$
\|H(b)+K\| \leq\|A+K\|+\|T(a)\| \leq\left(1+c_{p}\right)\|A+K\|,
$$

which completes the lower estimate. 
Corollary 18. For $1<p<\infty$, let $T$ and $H$ be forms of Toeplitz and Hankel type, respectively, and assume that $A=T+H$ is a compact operator on $H^{p}$. Then $T=C$ is a checkerboard form and $H=H(b)-C$, where $H(b)$ is a compact Hankel with $b \in C(\mathbb{T})$.

Proof. By Theorem 16 we can write $T=T(a)+C$ and $H=H(b)-C$ with $a, b \in L^{\infty}$ and $C$ a checkerboard form. Thus $A=T(a)+H(b)$, and Theorem 17 with $K=-A$ implies that $a=0$ and that $H(b)=-K$ is compact. The characterization of compact Hankel operators [3, Theorem 2.54] completes the proof.

Corollary 19. For $1<p<\infty$, let $A=T(a)+H$ where $a \in L^{1}$ and $H$ is a Hankel form.

(i) If $A$ is bounded on $H^{p}$, then $a \in L^{\infty}$ and $H=H(b)$ for some $b \in L^{\infty}$.

(ii) If $A$ is compact on $H^{p}$, then $a=0$ and $H=H(b)$ for some $b \in C(\mathbb{T})$.

Proof. In view of Theorem 16 and Corollary 18, it suffices to show that if $T(c)$ with $c \in L^{1}$ is a checkerboard form, then $c=0$. But this follows directly from the Riemann-Lebesgue lemma.

Finally, let us summarize the previous results by establishing equivalent characterizations of bounded Toeplitz + Hankel forms.

Theorem 20. Let $1<p<\infty$. Then the following statements are equivalent:

(i) A is a bounded linear operator on $H^{p}$ satisfying the relations

$$
\left\langle A \chi_{j-1}, \chi_{k}\right\rangle-\left\langle A \chi_{j}, \chi_{k-1}\right\rangle-\left\langle A \chi_{j}, \chi_{k+1}\right\rangle+\left\langle A \chi_{j+1}, \chi_{k}\right\rangle=0
$$

for all $j, k \in \mathbb{N}$.

(ii) $A$ is a bounded linear operator on $H^{p}$ which is a sum of a Toeplitz and a Hankel form.

(iii) $A=T(a)+H(b)$ for some $a, b \in L^{\infty}$.

Furthermore, the functions $a$ and $P\left(\chi_{-1} b\right)$ are uniquely determined, and

$$
\begin{aligned}
\|T(a)+H(b)\| & \simeq \max \left\{\|a\|_{\infty},\|H(b)\|\right\}, \\
\|T(a)+H(b)\|_{\mathrm{ess}} & \simeq \max \left\{\|a\|_{\infty},\|H(b)\|_{\mathrm{ess}}\right\},
\end{aligned}
$$

where " $\simeq "$ stands for equivalence of norms.

Proof. Similar to the proof of Theorem 10.

Elaborating on the last part of the previous theorem, we remark that the norm and the essential norm of a Hankel operator $H(b)$ on $H^{p}$ is equivalent 
to

$$
\operatorname{dist}_{L^{\infty}}\left(b, \overline{H^{\infty}}\right) \quad \text { and } \operatorname{dist}_{L^{\infty}}\left(b, C(\mathbb{T})+\overline{H^{\infty}}\right),
$$

respectively. For details see [3, Theorems 2.11 and 2.54] and the comments and references given there.

Acknowledgements. This project has received funding from the European Union's Horizon 2020 research and innovation programme under the Marie Sklodowska-Curie grant agreement No 844451. The authors also thank the American Institute of Mathematics and the SQuaRE program for their support. Virtanen was supported in part by EPSRC grant EP/T008636/1.

\section{REFERENCES}

[1] E. L. Basor and T. Ehrhardt, Fredholm and invertibility theory for a special class of Toeplitz + Hankel operators, J. Spectr. Theory 3 (2013), no. 2, 171-214.

[2] E. L. Basor and T. Ehrhardt, Asymptotic formulas for determinants of a special class of Toeplitz + Hankel matrices, in: Large truncated Toeplitz matrices, Toeplitz operators, and related topics, D. Bini, T. Ehrhardt, A. Karlovich, I. Spitkovsky (eds), Oper. Theory Adv. Appl. 259, Birkhäuser/Springer, Cham, 2017, 125-154.

[3] A. Böttcher and B. Silbermann, Analysis of Toeplitz Operators, 2nd Edition, Springer Verlag, Berlin, Heidelberg, New York, 2006.

[4] A. Brown and P. R. Halmos, Algebraic properties of Toeplitz operators, J. Reine Angew. Math. 213 (1963/64), 89-102.

[5] P. Deift, A. Its and I. Krasovsky, Asymptotics of Toeplitz, Hankel and Toeplitz+Hankel determinants with Fisher-Hartwig singularities, Ann. of Math. 174 (2011), no. 2, 1243-1299.

[6] P. Deift, A. Its and I. Krasovsky, Toeplitz matrices and Toeplitz determinants under the impetus of the Ising model: some history and some recent results, Comm. Pure Appl. Math. 66 (2013), no. 9, 1360-1438.

[7] V. D. Didenko and B. Silbermann, Invertibility and inverses of Toeplitz plus Hankel operators, J. Operator Theory 78 (2017), no. 2, 293-307.

[8] V. D. Didenko and B. Silbermann, Invertibility Issues for Toeplitz plus Hankel Operators and Their Close Relatives, arXiv: 2003.09115.

[9] R. V. Duduchava, The discrete Wiener-Hopf equations, Trudy Tbilis. Mat. Inst., 50, 42-59 (1975) (Russian).

[10] R. Gharakhloo and A. Its, A Riemann-Hilbert approach to asymptotic analysis of Toeplitz+Hankel determinants, to appear in Symmetry Integr. Geom., arXiv:1909.00963. 
[11] P. R. Halmos, A Hilbert Space Problem Book, Second edition, Graduate Texts in Mathematics, Vol. 19, and Encyclopedia of Mathematics and its Applications, Vol. 17, Springer, New York and Berlin (1982).

[12] P. Hartman, On completely continuous Hankel matrices, Proc. Amer. Math. Soc. 9 (1958), 862-866.

[13] G. Heinig and K. Rost, Matrices with displacement structure, generalized Bezoutians, and Moebius transformations, in: The Gohberg anniversary collection, H. Dym, S. Goldberg, M.A. Kaashoek, P. Lancaster (eds), Oper. Theory Adv. Appl. 40/41, Birkhäuser, Basel, 1989, 203230.

[14] G. Heinig and K. Rost, Inversion of matrices with displacement structure, Integr. Equat. Oper. Th. 12 (1989), no. 6, 813-834.

[15] B. Hollenbeck and I. E. Verbitsky, Best constants for the Riesz projection, J. Funct. Anal. 175 (2000), 370-392.

[16] S. Janson, J. Peetre and S. Semmes, On the action of Hankel and Toeplitz operators on some function spaces, Duke Math. J. 51 (1984), no. 4, 937-958.

[17] M. Lindner, Infinite Matrices and Their Finite Sections, Birkhäuser Verlag, Basel, Boston, Berlin, 2006.

[18] Z. Nehari, On bounded bilinear forms, Ann. of Math. (2) 65 (1957), 153-162.

[19] M. Papadimitrakis and J.A. Virtanen, Hankel and Toeplitz transforms on $H^{1}$ : continuity, compactness and Fredholm properties, Integr. Equat. Oper. Th. 61 (2008), no. 4, 573-591.

[20] V. Peller, Hankel operators and their applications, Springer Monographs in Mathematics. Springer-Verlag, New York, 2003.

[21] E. Shargorodsky, On the essential norms of Toeplitz operators with continuous symbols, arXiv: 2007.13178.

[22] O. Toeplitz, Zur Theorie der quadratischen und bilinearen Formen von unendlichvielen Veränderlichen, Math. Ann. 70 (1911), no. 3, 351-376.

[23] V. A. Tolokonnikov, Hankel and Toeplitz operators in Hardy spaces, Investigations on linear operators and the theory of functions XIV, Zap. Nauchn. Sem. Leningrad. Otdel. Mat. Inst. Steklov. (LOMI) 141 (1985), 165-175; English transl.: J. Sov. Math. 37 (1987), 1359-1364.)

[24] H. Widom, Asymptotic behavior of block Toeplitz matrices and determinants. II, Advances in Math. 21 (1976), no. 1, 1-29. 
Mathematics Department, University of California, 1156 High Street, Santa Cruz CA 95064, USA

Email address: tehrhard@ucsc.edu

Department of Mathematics, University of Reading, Whiteknights CamPUs, READing RG6 6AX, United Kingdom

Email address: r.t.hagger@reading.ac.uk

Department of Mathematics, Whiteknights Campus University of Reading, Reading RG6 6AX, United Kingdom, and, Department of Mathematics, University of Helsinki, Helsinki 00014, Finland

Email address: j.a.virtanen@reading.ac.uk, jani.virtanen@helsinki.fi 